\title{
Supporting Figures
}
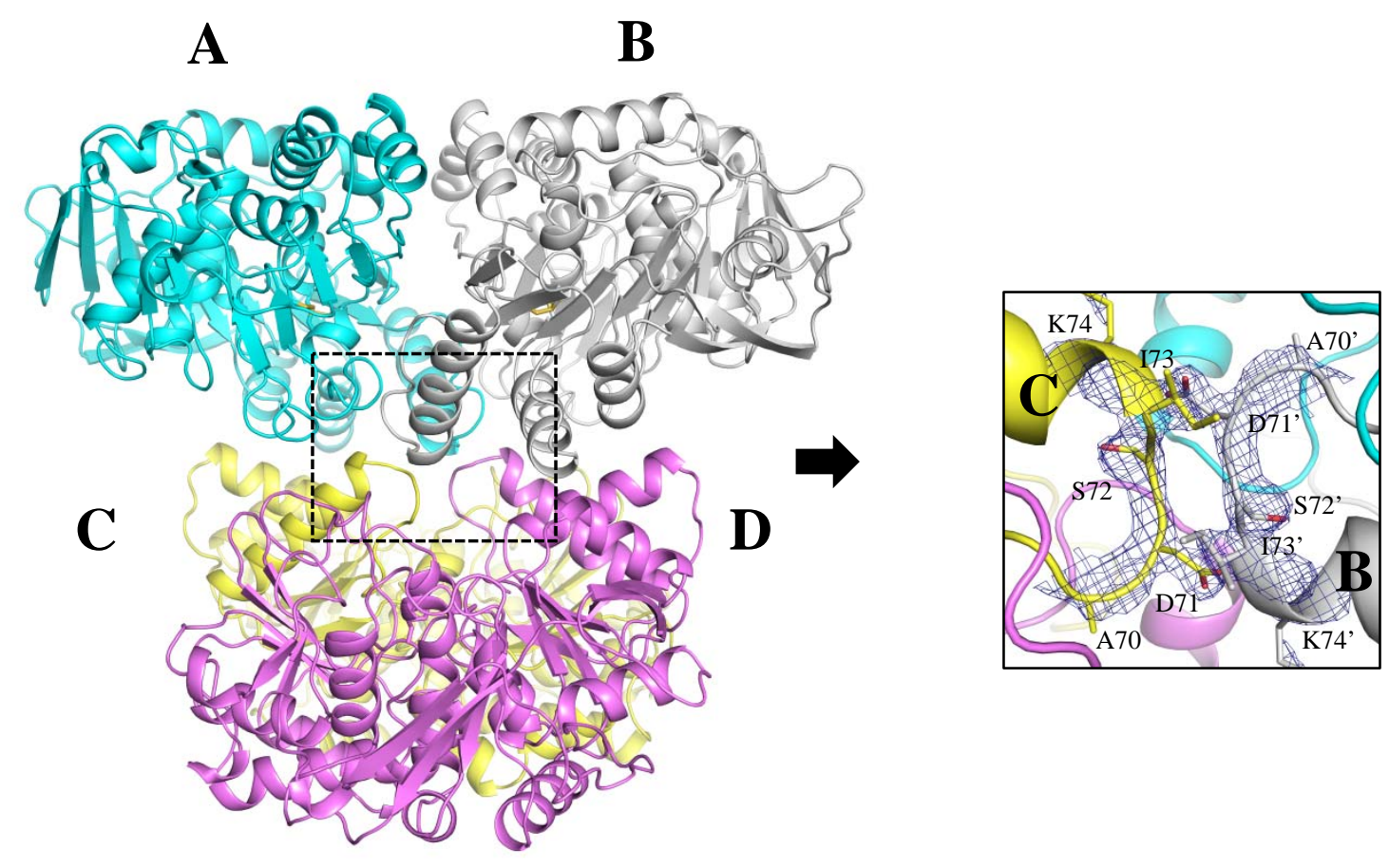

Supporting Figure S1 Crystal packing of ox-MJ0499. The 4 molecules of ox-MJ0499 show close contact in the crystal and form 2 homodimers (AB and CD). The molecules B and C shows close contact, as represented in the box on the right, the 2fo-fc map of A70 to K74 contoured at $1.5 \sigma$. The electron density of D71 side chain is not clearly distinguished from the neighboring I73' residue. 


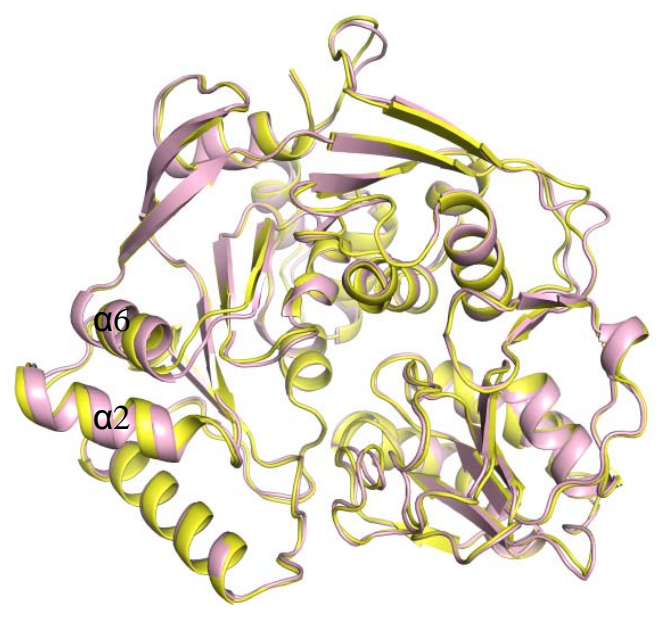

(a)

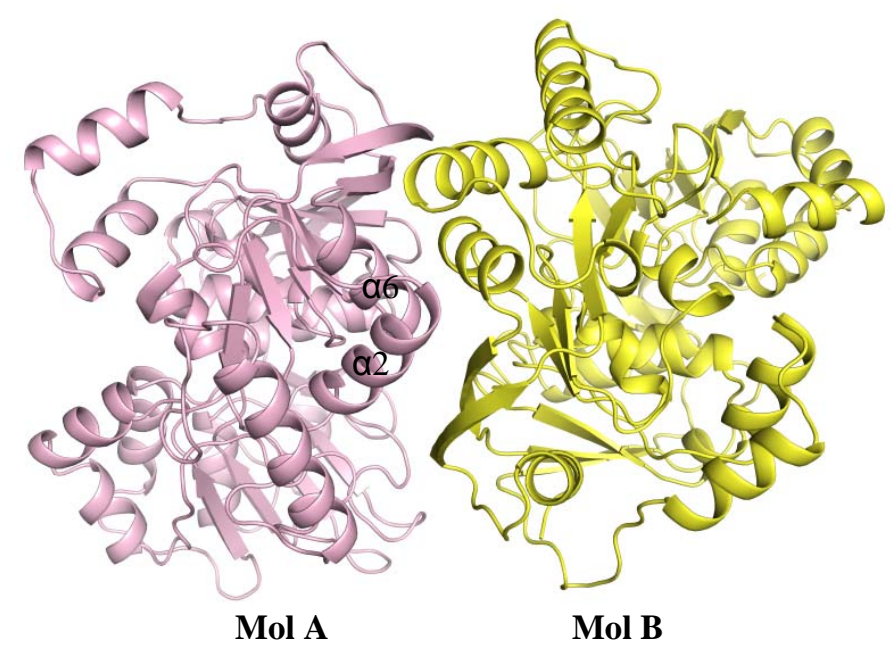

(b)

Supporting Figure S2 (a) Asymmetric unit of MJ1003. The 2 molecules of MJ1003 in an asymmetric unit are superposed upon each other. The helices $\alpha 2$ and $\alpha 6$ show structural differences. (b) Crystallographic dimer observed in an asymmetric unit. The 2 chains of MJ1003, MolA and MolB, form a crystallographic dimer and $\alpha 2$ and $\alpha 6$ helices contribute to dimer interaction. 


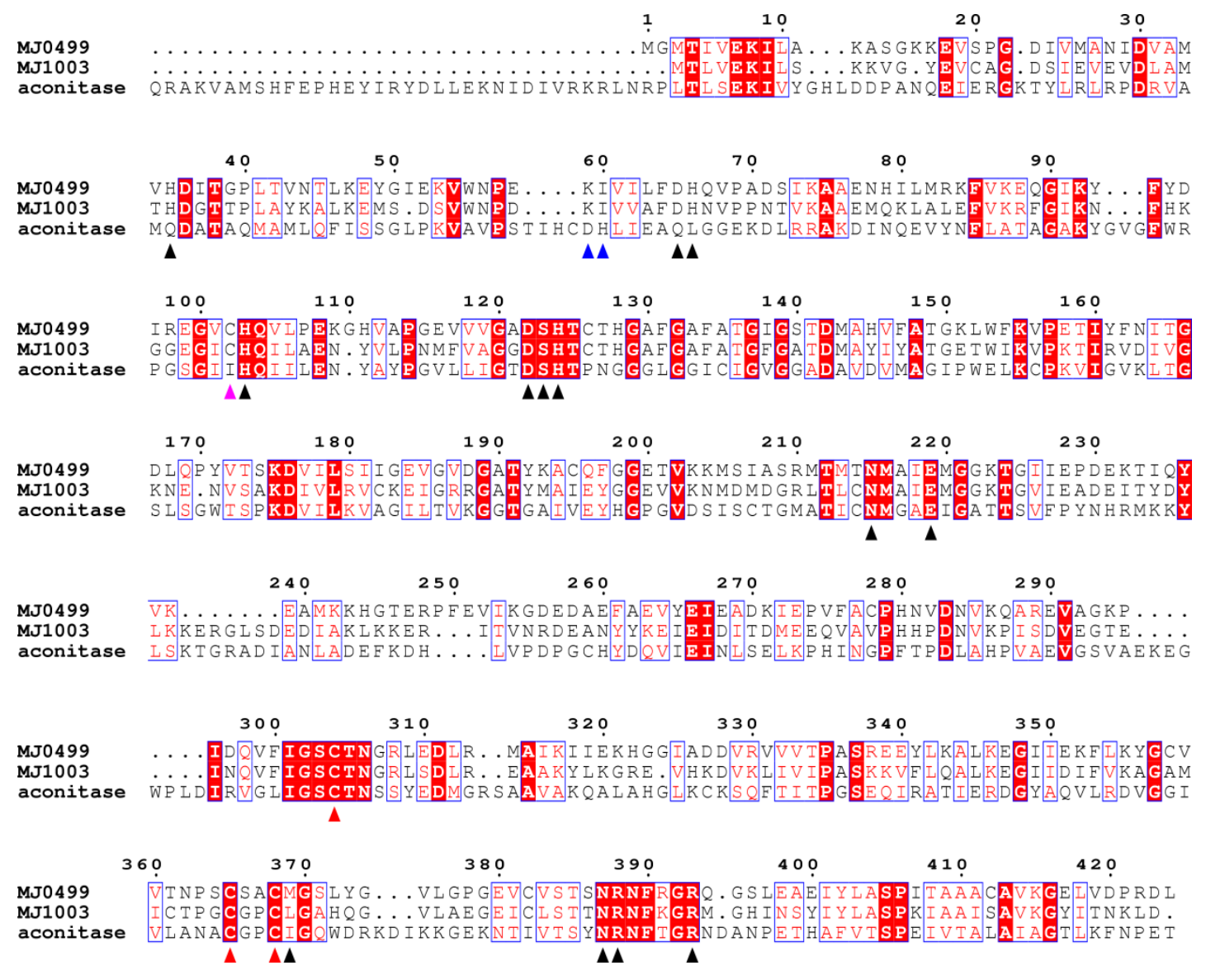

Supporting Figure S3 Multiple sequence alignment of aconitase family proteins. MJ0499 and MJ1003 from Methanococcus jannaschii, and mitochondrial aconitase from Sus scrofa (PDB entry 5acn). The conserved residues are shaded red and similar residues are displayed in red letters. Residues of active site residues are indicated by black triangles below the aligned sequence and three cysteine residues involved in iron-sulfur cluster binding are indicated by red triangles. The fourth cysteine residue is indicated by a pink triangle. The two residues in aconitase indicated by blue triangles, D100 and H101, align only in the conserved sequences. 


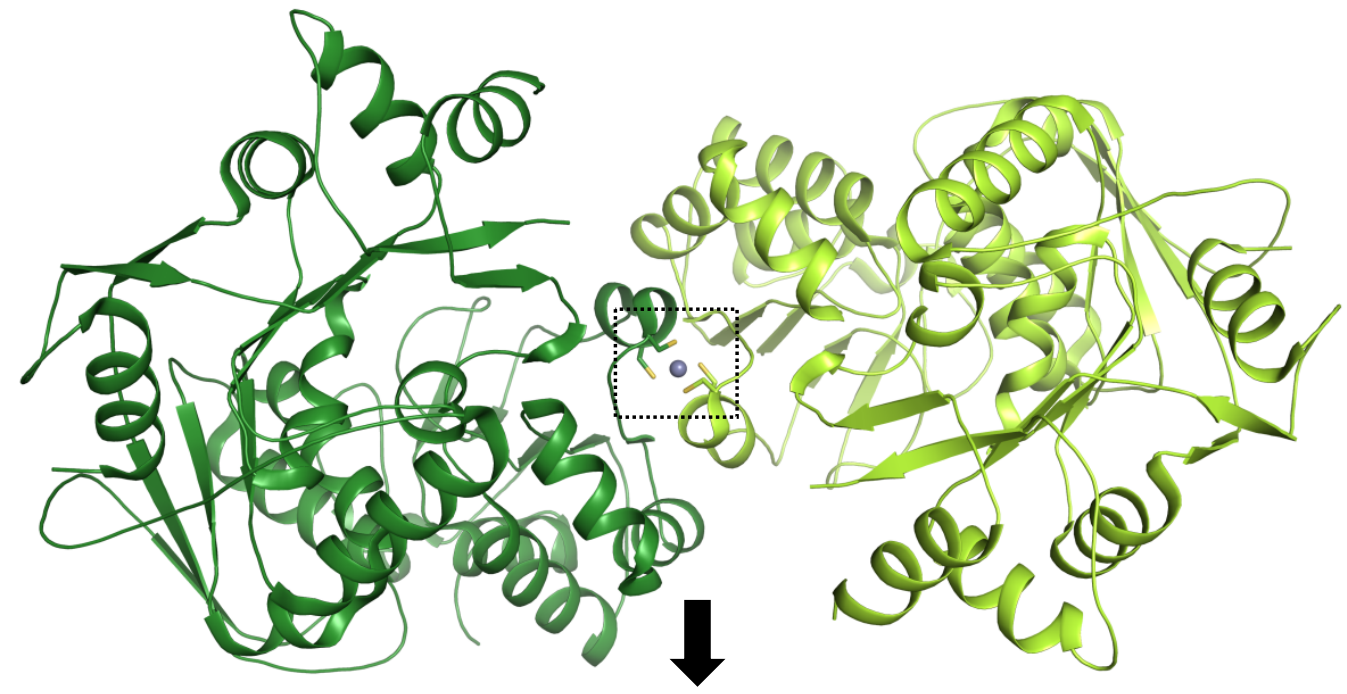

Mol A

Mol B

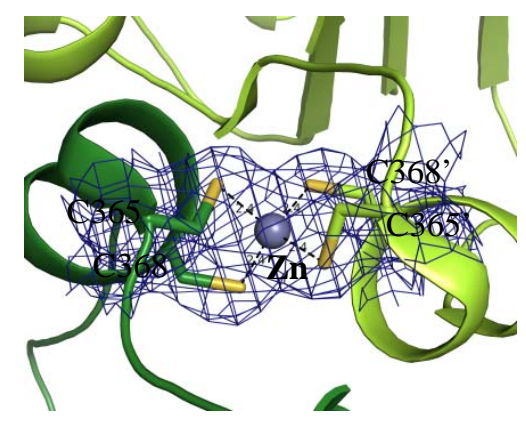

Supporting Figure S4 Molecular interaction in the crystal of red-MJ0499. The C365 and C368 residues coordinate an zinc ion (Zn) with the two symmetrical Cys residues from the other molecule. The average distance of zinc ion coordination is $2.4 \AA$. The interface is shown magnified in the square box and the 2fo-fc map is shown at $1.5 \sigma$ cut-off. 


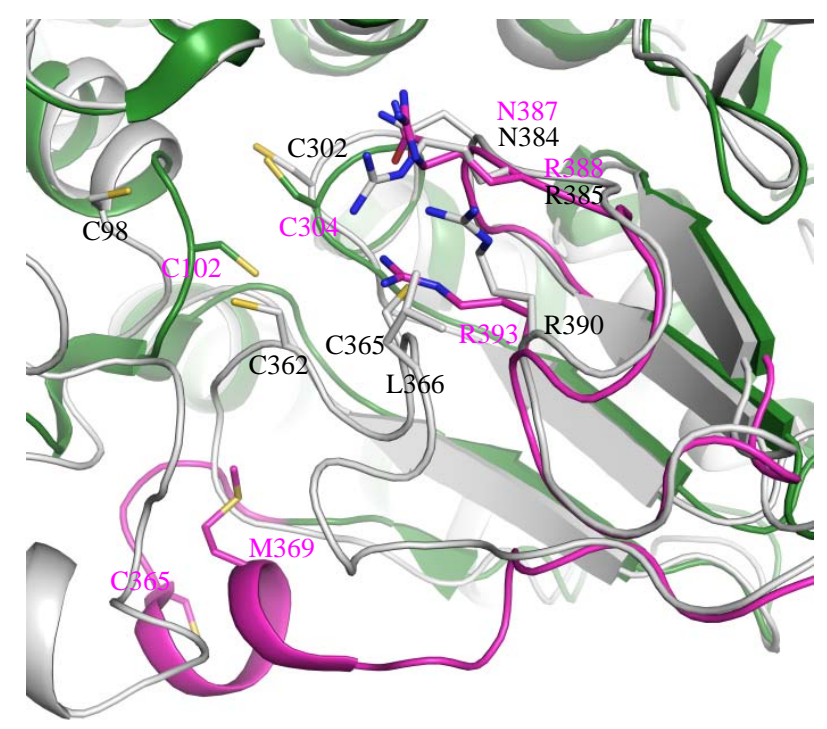

Supporting Figure S5 The active site comparison of red-MJ0499 (green) and MJ1003 (gray). The regions showing conformational change upon reduction are indicated in red-MJ0499 in magenta color. The active site residues are represented as stick models and residues are labeled in different colors, magenta for red-MJ0499 and black for MJ1003. 


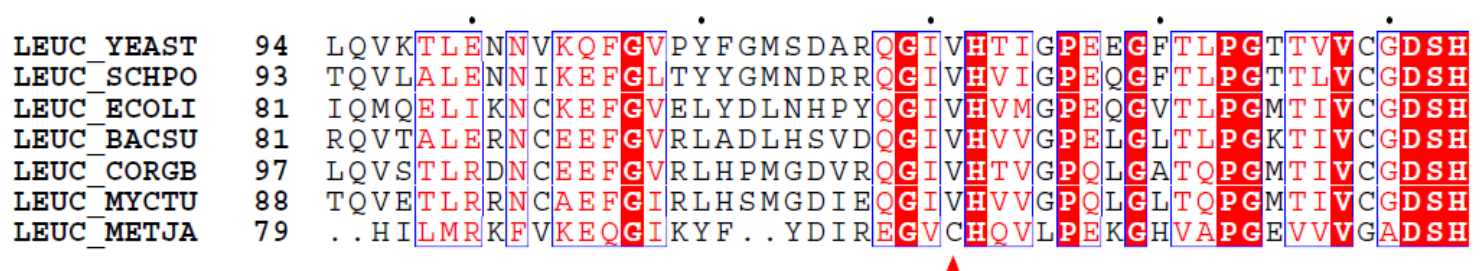

(a)

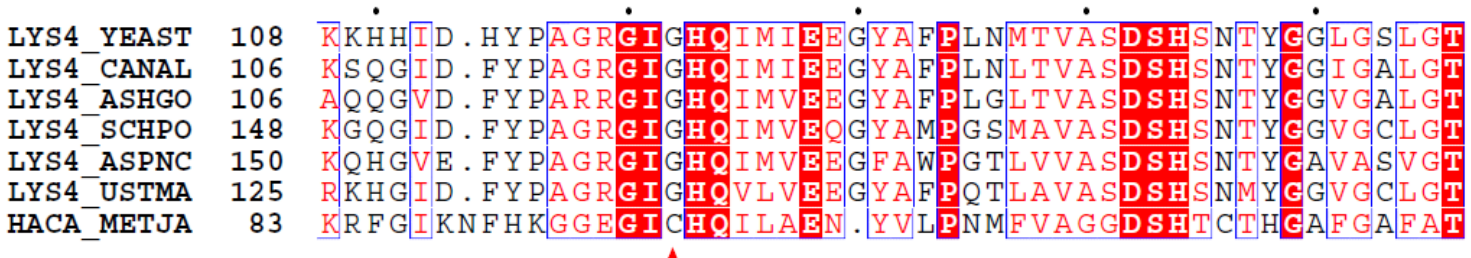

(b)

Supporting Figure S6 (a) Multiple sequence alignments of IPM isomerases. The partial sequence alignments are represented and the fourth cysteine is indicated by a red triangle. LEUC_YEAST, 3-isopropylmalate dehydratase from Saccharomyces cerevisiae; LEUC_SCHPO, 3-isopropylmalate dehydratase from Schizosaccharomyces pombe; LEUC_ECOLI, 3-isopropylmalate dehydratase large subunit from Escherichia coli; LEUC_BACSU, 3-isopropylmalate dehydratase large subunit from Bacillus subtilis; LEUC_CORGB, 3-isopropylmalate dehydratase large subunit from Corynebacterium glutamicum; LEUC_MYCTU, 3-isopropylmalate dehydratase large subunit from Mycobacterium tuberculosis; LEUC_METJA (MJ0499), Isopropylmalate/citramalate isomerase large subunit from Methanococcus jannaschii. (b) Multiple sequence alignments of homoaconitases. The partial sequence alignments are represented and the fourth cysteine is indicated by a red triangle. LYS4_YEAST, homoaconitase from Saccharomyces cerevisiae; LYS4_CANAL, homoaconitase from Candida albicans; LYS4_ASHGO, homoaconitase from Ashbya gossypii; LYS4_SCHPO, homoaconitase from Schizosaccharomyces pombe; LYS4_ASPNC, homoaconitase from Aspergillus niger; LYS4_USTMA, homoaconitase from Ustilago maydis; HACA_METJA (MJ1003), Homoaconitase large subunit from Methanocaldococcus jannaschii. 